\title{
Enhanced GSA-Based Optimization for Minimization of Power Losses in Power System
}

\author{
Gonggui Chen, ${ }^{1,2,3}$ Lilan Liu, ${ }^{1}$ and Shanwai Huang ${ }^{1}$ \\ ${ }^{1}$ Key Laboratory of Industrial Internet of Things and Networked Control, Ministry of Education, \\ Chongqing University of Posts and Telecommunications, Chongqing 400065, China \\ ${ }^{2}$ Research Center on Complex Power System Analysis and Control, Chongqing University of Posts and Telecommunications, \\ Chongqing 400065, China \\ ${ }^{3}$ Department of Electrical Engineering, Hubei Minzu University, Enshi 445000, China
}

Correspondence should be addressed to Gonggui Chen; chenggpower@126.com

Received 14 July 2015; Revised 24 November 2015; Accepted 1 December 2015

Academic Editor: Luis J. Yebra

Copyright (C) 2015 Gonggui Chen et al. This is an open access article distributed under the Creative Commons Attribution License, which permits unrestricted use, distribution, and reproduction in any medium, provided the original work is properly cited.

Gravitational Search Algorithm (GSA) is a heuristic method based on Newton's law of gravitational attraction and law of motion. In this paper, to further improve the optimization performance of GSA, the memory characteristic of Particle Swarm Optimization (PSO) is employed in GSAPSO for searching a better solution. Besides, to testify the prominent strength of GSAPSO, GSA, PSO, and GSAPSO are applied for the solution of optimal reactive power dispatch (ORPD) of power system. Conventionally, ORPD is defined as a problem of minimizing the total active power transmission losses by setting control variables while satisfying numerous constraints. Therefore ORPD is a complicated mixed integer nonlinear optimization problem including many constraints. IEEE14bus, IEEE30-bus, and IEEE57-bus test power systems are used to implement this study, respectively. The obtained results of simulation experiments using GSAPSO method, especially the power loss reduction rates, are compared to those yielded by the other modern artificial intelligence-based techniques including the conventional GSA and PSO methods. The results presented in this paper reveal the potential and effectiveness of the proposed method for solving ORPD problem of power system.

\section{Introduction}

Optimal reactive power dispatch (ORPD), as one of the significant optimization problems in power system operation, is to minimize the given objective function such as total active power transmission losses $\left(P_{\text {Loss }}\right)$ by optimizing settings of control variables while satisfying a set of constraints during the entire dispatch period. Control variables contain discrete variables such as tap positions of transformers and amount of reactive compensation and continuous variables like generator voltages. Constraints consist of a series of equality constraints and inequality constraints [1]. Besides, it is worth noting that the ORPD problem in this paper is a single-objective optimization problem different from the study in [1] which researches a multiobjective optimization problem. In [1], the multiobjective ORPD problem seeks for a compromise solution for minimization of power losses and $L$ index simultaneously, but this paper is required to find out a global optimal solution for minimization of $P_{\text {Loss }}$. Thus, ORPD problem in this paper is a complex mixed integer nonlinear optimization problem with a number of constraints and has the challenge of searching for the global optimal solution.

Numerous classical approaches including gradient-based optimization algorithms and many mathematical programming methods [2-5] have been developed and applied for solving the ORPD problem in the past. However, these traditional techniques almost only optimize the differentiable objective functions and they have difficulties in dealing with the nonconvex, nonlinear, discontinuous functions with constraints $[6,7]$. But now a number of modern artificial intelligence-based techniques with stochastic optimization such as Genetic Algorithm (GA) [8], Differential Evolution (DE) [9], Particle Swarm Optimization (PSO) [10], and Gravitational Search Algorithm (GSA) have been applied to solve different ORPD problems efficiently without the 
abovementioned restraints, which overcomes the defects of conventional techniques. Furthermore, different method has its peculiar strength; for instance, the process of variation and hybridization in GA increases the diversity of population, which contributes to obtain the better solutions; DE uses the differences between individuals to change the individual itself, and this operation utilizes the distribution features of population to improve the search capacity effectively; PSO gets highlighted by virtue of the memory characteristic from imitating animals' predation process containing social and individual behaviors; the pattern of movement of GSA contributes significantly to the high efficiency of the search process. Conversely, every method yet has its own weakness: GA is apt to converge prematurely and the long and complicated evolution procedures of it increase the running time; in DE, the individuals' differences decrease with the increase of number of iterations, which impacts the increase of diversity of population directly. PSO tends to be trapped into prematurity in the latter period of searching, which directly lessens the possibility of acquiring the better solution; different from the algorithms based on biology, GSA is a memoryless algorithm, which is adverse to recording of the optimal value during the process of searching. According to these features, the exertion of merging individual superiorities into a new algorithm has become wide now in various engineering fields, which avoids their own disadvantages by benefiting from each other's advantages; for example, a method composed of chaotic embedded Backtracking Search Optimization Algorithm (BSA) and Binary Charged System Search (BCSS) algorithm is proposed for solving Short-Term Hydrothermal Generation Scheduling (SHTGS) in [11]; in [12], the authors present a new hybrid evolutionary algorithm based on new fuzzy adaptive PSO algorithm and NelderMead simplex search method to solve distribution feeder reconfiguration problem; the combination of the vertical search algorithm and presented lateral search algorithm is used to solve the midterm schedule for thermal power plants problem in [13]. But this paper highly favors the superiorities of PSO and GSA. On the basis of GSA, PSO is merged with it as fine tuning to improve the quality of solutions, which forms GSAPSO method for solving ORPD problem.

Gravitational Search Algorithm (GSA), a heuristic evolutionary optimization algorithm, was proposed by Rashedi et al. in [14]. GSA deriving from the thought of Newton's law stands out depending on the flexible and efficient movement characteristic, and naturally, its application is wide. GSAbased Photovoltaic (PV) excitation control strategy is used for single-phase operation of three-phase wind-turbine coupled induction generator [15]. GSA is applied to coordinate Power System Stabilizers (PSSs) and Thyristor Controlled Series Capacitor (TCSC) controllers simultaneously, which is demonstrated to achieve good robust performance for damping the low frequency interarea oscillations [16]. GSA is proposed to find the optimal solution for optimal power flow problem in a power system [17].

Particle Swarm Optimization (PSO) proposed by Kennedy and Eberhart [18] owns numerous absorbing aspects: simple thought, convenient implementation, high efficiency, powerful search ability, and so on. But what the most prominent feature is belongs to the memory peculiarity during the search process, the memory peculiarity contributing particles to record the global optimum and individual optimum in every generation. The applications of PSO are far more than the authors care to mention; for instance, enhanced PSO approach is applied for optimal scheduling of hydrosystem [19]; robust PID controller tuning based on the constrained PSO is researched in [20]; PSO is used for optimization of acoustic filters [21]; a hybrid Particle Swarm Optimization algorithm is proposed for solving the problem of optimal reactive power dispatch within a wind farm [10].

In this paper, GSAPSO is the combination of GSA and PSO, which not only retains movement characteristic in the search process of GSA but also increases capability of sharing information and memory ability. In this work, GSA, PSO, and GSAPSO have been examined and tested in IEEE14-bus, IEEE30-bus, and IEEE57-bus test systems for the solution of ORPD problem of power system with the objective minimizing total active power transmission losses $\left(P_{\text {Loss }}\right)$. The obtained performances of GSA, PSO, and GSAPSO are compared. And the power loss reduction rates of the proposed GSAPSO algorithm are also compared to those of other optimization methods. The simulation results reveal that the proposed GSAPSO approach can obtain a better optimum effect than these compared algorithms and the results' distribution of it is more concentrated than conventional GSA and PSO methods; besides, the proposed GSAPSO can avoid falling into the local optimum.

The rest of this paper is organized as follows: Section 2 introduces the mathematical modeling of ORPD problem. GSAPSO algorithm is described in detail in Section 3. Section 4 presents the calculation process of GSAPSO algorithm for ORPD problem. Some simulation experiments are shown in Section 5, and Section 6 gives the conclusions.

\section{Mathematical Modeling}

The mathematical modeling of ORPD problem is composed of two parts: objectives and constraints. The former provided in the paper are to minimize $P_{\text {Loss }}$, and the latter contain equality constraints and inequality constraints [1].

2.1. Objective Functions. The objective to minimize the total active power transmission losses in reactive power optimization is expressed as follows:

$$
\begin{aligned}
& \min f \\
& =\min \left\{P_{\text {Loss }}=\sum_{k \in N_{P}} g_{k}\left(V_{i}^{2}+V_{j}^{2}-2 V_{i} V_{j} \cos \delta_{i j}\right)\right\},
\end{aligned}
$$

where $P_{\text {Loss }}$ represents the total active power losses in transmission lines; $N_{P}$ is the number of network branches; $g_{k}$ is the conductance of the $k$ th branch which connects bus $i$ and bus $j ; V_{i}$ and $V_{j}$, respectively, denote the voltage magnitude of the $i$ th and $j$ th bus; $\delta_{i j}$ is the voltage phase between buses $i$ and $j$. 
2.2. System Constraints. The aforementioned objective function is subject to the system constraints which include the equality and inequality constraints.

2.2.1. Equality Constraints. There are two equality constraints describing the active and reactive power balance, which are expressed as follows:

$$
\begin{aligned}
P_{g i}-P_{l i}-V_{i} \sum_{j=1}^{n} V_{j}\left(G_{i j} \cos \delta_{i j}+B_{i j} \sin \delta_{i j}\right)=0 & \quad i \in N \\
Q_{g i}-Q_{l i}-V_{i} \sum_{j=1}^{n} V_{j}\left(G_{i j} \cos \delta_{i j}-B_{i j} \sin \delta_{i j}\right)= & \\
& \quad i \in N_{\mathrm{PQ}}
\end{aligned}
$$

where $n$ is the number of the buses connecting with the $i$ th bus; $N$ is the number of total buses except for swing bus; $N_{\mathrm{PQ}}$ is the number of PQ buses; $P_{g i}$ and $P_{l i}$ are the active power of the $i$ th generator bus and the $i$ th load bus, respectively; $Q_{g i}$ and $Q_{l i}$ are, respectively, the reactive power at the $i$ th generator bus and the $i$ th load bus; $G_{i j}$ and $B_{i j}$, respectively, represent the real part and imaginary part of $Y_{i j}$ which is the element of the $Y$-bus matrix (bus admittance matrix) at the $i$ th row and the $j$ th column.

Equations (2) are considered as the termination conditions of calculating the Jacobian matrix in Newton-Raphson load flow calculation.

2.2.2. Inequality Constraints. The descriptions of inequality constraints are given based on the state variables and control variables, respectively.

\section{(i) Inequality Constraints of Control Variables}

(a) The limit for generator bus voltages:

$$
V_{g i}^{\min } \leq V_{g i} \leq V_{g i}^{\max }, \quad i \in N_{\mathrm{PV}}
$$

where $N_{\mathrm{PV}}$ denotes the number of PV buses and $V_{g i}$ is the voltages at the $i$ th generator bus.

(b) The limit for tap positions of transformers:

$$
T_{i}^{\min } \leq T_{i} \leq T_{i}^{\max }, \quad i \in N_{T}
$$

where $N_{T}$ is the number of transformers; $T_{i}$ is the tap positions of the $i$ th transformer, which is a discrete variable.

(c) The limit for amount of reactive compensation:

$$
Q_{c i}^{\min } \leq Q_{c i} \leq Q_{c i}^{\max }, \quad i \in N_{C}
$$

where $N_{C}$ is the number of the banks of capacitor or inductor; $Q_{c i}$ denotes the reactive compensation capacity of the $i$ th bank of capacitor or inductor. (ii) Inequality Constraints of State Variables

(a) The limit for voltages at PQ bus:

$$
V_{l i}^{\min } \leq V_{l i} \leq V_{l i}^{\max }, \quad i \in N_{\mathrm{PQ}},
$$

where $V_{l i}$ is the voltage at the $i$ th load bus.

(b) The limit for reactive compensation capacity at PV bus:

$$
Q_{g i}^{\min } \leq Q_{g i} \leq Q_{g i}^{\max }, \quad i \in N_{\mathrm{PV}},
$$

where $Q_{g i}$ is the reactive compensation capacity at the generator $i$.

(c) The limit for apparent power of transmission line:

$$
S_{i j} \leq S_{i j}^{\max }, \quad i j \in N_{P},
$$

where $S_{i j}$ is the apparent power of the transmission line between buses $i$ and $j$.

2.3. Handling of Constraints. What is worth mentioning is that, during the process of optimization, equality constraints and inequality constraints of control and state variables are satisfied as the following explanations, respectively.

(i) The two equality constraints are satisfied by NewtonRaphson power flow algorithm in load flow calculation.

(ii) The generator bus voltages $\left(V_{g i}\right)$, tap positions of transformers $\left(T_{i}\right)$, and amount of reactive compensation $\left(Q_{c i}\right)$ are the control variables which can be selfrestricted according to their limits by the algorithm.

(iii) The limits on active power generation at the swing bus $\left(P_{\text {gswing }}\right)$, voltages at PQ bus $\left(V_{l i}\right)$, reactive compensation capacity at PV bus $\left(Q_{g i}\right)$, and apparent power of transmission line $\left(S_{i j}\right)$ are state variables which are restricted by the objective function combining the penalty function.

2.4. Formulation. In short, the ORPD problem can be formulated as a complex nonlinear constrained optimization mathematical model, and a compact expression is given in

$$
\begin{array}{ll}
\min & f=P_{\text {Loss }} \\
\text { s.t. } & G(c, s)=0 \\
& H(c, s) \leq 0 \\
& c_{\min } \leq c \leq c_{\max } \\
& s_{\min } \leq s \leq s_{\max },
\end{array}
$$

where $c$ and $s$ denote the vector of control variables and the vector of state variables, respectively; $G(c, s)$ and $H(c, s)$, respectively, represent the equality constraints and inequality constraints of system.

In this paper, $c$ and $s$ are expressed as follows:

$$
\begin{aligned}
c^{T} & =\left[V_{g 1}, \ldots, V_{g N_{\mathrm{PV}}}, T_{1}, \ldots, T_{N_{T}}, Q_{c 1}, \ldots, Q_{c N_{C}}\right], \\
s^{T} & =\left[V_{l 1}, \ldots, V_{l N_{\mathrm{PQ}}}, Q_{g 1}, \ldots, Q_{g N_{\mathrm{PV}}}, S_{i j 1}, \ldots, S_{i j N_{P}}\right],
\end{aligned}
$$

where " $T$ " denotes transposition. 


\section{Description of GSAPSO Algorithm}

3.1. Brief Introduction of GSA. GSA proposed by Rashedi et al. in 2009 is a newly developed stochastic search algorithm which is inspired by the law of gravity and law of motion [14]. In GSA, a series of agents are considered as objects and their performances are measured by their masses, and all these objects attract each other by the gravity force, while this force causes a global movement of all objects towards the objects with heavier masses [22]. The description of GSA about how to solve the problem is as follows.

Assume there are $N$ agents distributed in space and the position of the $i$ th agent is defined as in

$$
X_{i}=\left(x_{i}^{1}, \ldots, x_{i}^{d}, \ldots, x_{i}^{D}\right) \quad i=1,2, \ldots, N,
$$

where $x_{i}^{d}$ denotes the position of the $i$ th agent in the $d$ th dimension and $D$ is the dimension of the search space.

The mass of every agent is computed based on the current agents' fitness as follows:

$$
\begin{aligned}
m_{i}(k) & =\frac{\text { fit }_{i}(k)-f_{\text {worst }}(k)}{f_{\text {best }}(k)-f_{\text {worst }}(k)}, \\
M_{i}(k) & =\frac{m_{i}(k)}{\sum_{j=1}^{N} m_{j}(k)},
\end{aligned}
$$

where $M_{i}(k)$ and fit $i(k)$ represent the mass and fitness value of the $i$ th agent at iteration $k ; f_{\text {best }}(k)$ and $f_{\text {worst }}(k)$, respectively, denote the best and worst fitness value among the $N$ agents at iteration $k$, which is defined as follows:

$$
\begin{aligned}
f_{\text {best }}(k) & =\min _{i \in\{1, \ldots, N\}} \mathrm{fit}_{i}(k), \\
f_{\text {worst }}(k) & =\max _{i \in\{1, \ldots, N\}} \mathrm{fit}_{i}(k) .
\end{aligned}
$$

In accordance with the law of gravity, the force acting on agent $i$ by agent $j$ is computed as follows:

$$
F_{i j}^{d}(k)=G(k) \cdot \frac{M_{i}(k) \cdot M_{j}(k)}{R_{i j}(k)+\varepsilon} \cdot\left(x_{j}^{d}(k)-x_{i}^{d}(k)\right),
$$

where $G(k)$ is the gravitational constant at iteration $k ; \varepsilon$ represents a small constant which can avoid the denominator equal to zero; $R_{i j}(k)$, defined as $R_{i j}(k)=\left\|x_{i}(k), x_{j}(k)\right\|_{2}$, denotes the Euclidian distance between agent $i$ and agent $j$.

We always use $R_{i j}(k)$ rather than $R_{i j}(k)^{2}$ in (15) because of the better performance of $R_{i j}(k)$ in most cases based on many simulation experiments. The better performance refers to the lower power losses in this paper. And $G(k)$ is reduced from an initial value with iteration $k$ as follows:

$$
G(k)=G_{0} \cdot e^{-\alpha\left(k / k_{\max }\right)},
$$

where $G_{0}$ is the initial gravitational constant; $\alpha$ is a constant greater than zero; $k$ is the current number of iterations; and $k_{\text {max }}$ represents the maximum number of iterations.

On the basis of (15), the total force acting on agent $i$ can be given as

$$
F_{i}^{d}(k)=\sum_{j \in k_{\text {best }}, j \neq 1} \operatorname{rand} \cdot F_{i j}^{d}(k),
$$

where rand represents random number drawn uniformly on $(0,1) ; k_{\text {best }}$ denotes the set of the first $K$ agents with the best fitness value and biggest mass, which is a function and reduced with time from the initial value $K_{0}$.

Based on the law of motion, the acceleration of the $i$ th agent is computed as follows:

$$
a_{i}^{d}(k)=\frac{F_{i}^{d}(k)}{M_{i}(k)} .
$$

The updates of velocity and position of agent $i$ at the next iteration are computed as follows:

$$
\begin{aligned}
& v_{i}^{d}(k+1)=\operatorname{rand} \cdot v_{i}^{d}(k)+a_{i}^{d}(k), \\
& x_{i}^{d}(k+1)=x_{i}^{d}(k)+v_{i}^{d}(k+1),
\end{aligned}
$$

where $v_{i}^{d}(k)$ and $x_{i}^{d}(k)$ are the velocity and position of agent $i$ at iteration $k$ in the $d$ th dimension.

3.2. Memory Characteristic of PSO. Schools of fish and flocks of birds always find foods, which is attributed to the communication among individuals and the memory ability for individual best direction and global best direction. PSO algorithm simulates the behaviors of animals, whose update of velocity is defined as follows:

$$
\begin{aligned}
v_{i}^{d}(k+1)= & w \cdot v_{i}^{d}(k)+c_{1} \cdot \operatorname{rand} \cdot\left(P_{i \text { best }}^{d}-X_{i}^{d}(k)\right) \\
& +c_{2} \cdot \operatorname{rand} \cdot\left(P_{\text {gbest }}^{d}-X_{i}^{d}(k)\right),
\end{aligned}
$$

where $w$ is the inertia weight; $c_{1}$ and $c_{2}$ represent the acceleration factors; $P_{i \text { best }}^{d}$ and $P_{g d}^{d}$, respectively, denote the best position of particle $i$ and the best position in swarm in the $d$ th dimension at iteration $k$.

3.3. GSAPSO. Different from PSO, every agent in GSA determines the direction by the total force from other agents but lacks the communication with others so as to miss the memory ability. In this paper, the proposed GSAPSO is an enhanced GSA-based optimization algorithm, combining the memory characteristic of PSO based on the GSA, which is helpful to the agents to move to the global best position. The difference between GSA and GSAPSO is the update modes of velocity and position which are crucial for the artificial intelligence-based algorithms. And the updates of velocity and position in GSAPSO combining the law of gravity and memory characteristic of PSO are defined as follows:

$$
\begin{aligned}
v_{i}^{d}(k+1)= & \text { rand } \cdot v_{i}^{d}(k)+a_{i}^{d}(k)+c_{1} \cdot \text { rand } \\
& \cdot\left(P_{i \text { best }}^{d}-X_{i}^{d}(k)\right)+c_{2} \cdot \text { rand } \\
& \cdot\left(P_{\text {gbest }}^{d}-X_{i}^{d}(k)\right), \\
x_{i}^{d}(k+1)= & x_{i}^{d}(k)+v_{i}^{d}(k+1) .
\end{aligned}
$$

It is worth pointing out that the inertia weight impacting velocity in (20) is not introduced in (21), because GSA can 
determine the direction by the total force from other agents and only need to reinforce the impact of the memory for the best position of every agent and the best position in all the agents. And the steps of GSAPSO are depicted as follows.

Step 1. Generate the initial population.

Step 2. Compute the fitness of every agent, and record $P_{i \text { best }}^{d}$ and $P_{g d}^{d}$

Step 3. Update $G, f_{\text {best }}, f_{\text {worst }}$, and $M$ of the population based on (13), (14), and (16).

Step 4. Compute $F$ and $a$ of every agent according to (15), (17), and (18).

Step 5. Update the velocity and position by using (21) and (22).

Step 6 (check stop criterion). Go to the next step if the number of iterations reaches the maximum number of iterations; otherwise go back and continue Step 2.

Step 7. Select the solution with the best fitness as the global best solution.

And the computational flow of the GSAPSO algorithm is shown in Figure 1.

\section{The Calculation Process of GSAPSO Algorithm for ORPD Problem}

In the application of GSAPSO for solving the ORPD problem, the fitness value of every agent is the objective function value $\left(P_{\text {Loss }}\right)$, and the position of the agent is the solution which is a set of control variables containing the generator bus voltages, tap positions of transformers, and amount of reactive compensation. These control variables are applied in Newton-Raphson load flow calculation to obtain the total active power transmission losses, constraint violations of variables, reactive compensation capacity at PV bus, and so on. The optimization procedures of GSAPSO for ORPD problem are as follows.

Step 1. Set the parameters of power system and those of GSAPSO.

Step 2. Generate the initial population.

The vector of control variables expressed in (10) is as an agent to represent a potential solution for ORPD problem in GSAPSO. The initial population is made up of $N$ agents, and the $j$ th particle in the $d$ th dimension is generated based on

$$
\begin{aligned}
& c_{j, d}=c_{\min , d}+\operatorname{rand} \cdot\left(c_{\max , d}-c_{\min , d}\right) \\
& \qquad j \in[1, N], d \in[1, D],
\end{aligned}
$$

where $N$ is the size of population, $D$ is the dimension of control variables, and $N \times D$ can represent the dimension of the population. Besides, the initial velocity of population is set as a zero matrix with $N \times D$ dimension.

Step 3. Compute the objective function value $\left(P_{\text {Loss }}\right)$ of every agent by Newton-Raphson load flow calculation, and compare those of agents, and then record $P_{i \text { best }}^{d}$ and $P_{g d}^{d}$ as follows:

if

(i).Obj < $\mathrm{pBJ}(i) . \mathrm{Obj}$;

$\%(i)$.Obj represents the objective value of agent $i$

$\mathrm{pBJ}(i) \cdot$ Position $=p(i) \cdot$ Positon;

$\% \mathrm{pBJ}(i)$.Position represents the $P_{i \text { best }}^{d}$

end

if

gB.Obj < gBJ.Obj

$\mathrm{gBJ}(i)$.Position = gB.Positon;

$\% \mathrm{gBJ}(i)$.Position represents the $P_{g d}^{d}$

end

Step 4. Update $G, f_{\text {best }}, f_{\text {worst }}$, and $M$ of the population based on (13), (14), and (16).

Step 5. Compute $F$ and $a$ of every agent according to (15), (17), and (18).

Step 6. Update the velocity and position by using (21) and (22), and generate a new population.

Step 7 (check stop criterion). Go to the next step if the number of iterations reaches the maximum number of iterations; otherwise go back and continue Step 2.

Step 8. The solution with the best fitness (the lowest $P_{\text {Loss }}$ ) is the global best solution.

\section{Simulation Experiments}

In order to verify the effectiveness of the proposed GSAPSO algorithm compared to the traditional GSA and PSO, they have been examined and tested, respectively, in three IEEE test systems to solve the ORPD problem of power system with the objective minimizing total active power transmission losses $\left(P_{\text {Loss }}\right)$. The system data of test systems is depicted in Table 1. All the code of the abovementioned algorithms is written by MATLAB R2013b programming language and run on PC with Intel(R) Core 2 Duo CPU E7500 @ $2.93 \mathrm{GHz}$ with 2 GB RAM.

\subsection{Descriptions of Test Systems}

5.1.1. IEEE14-Bus System. The IEEE14-bus test system is taken from [23], whose single-line diagram is shown in Figure 2. The reactive power limits of generators are given in Table 2, 


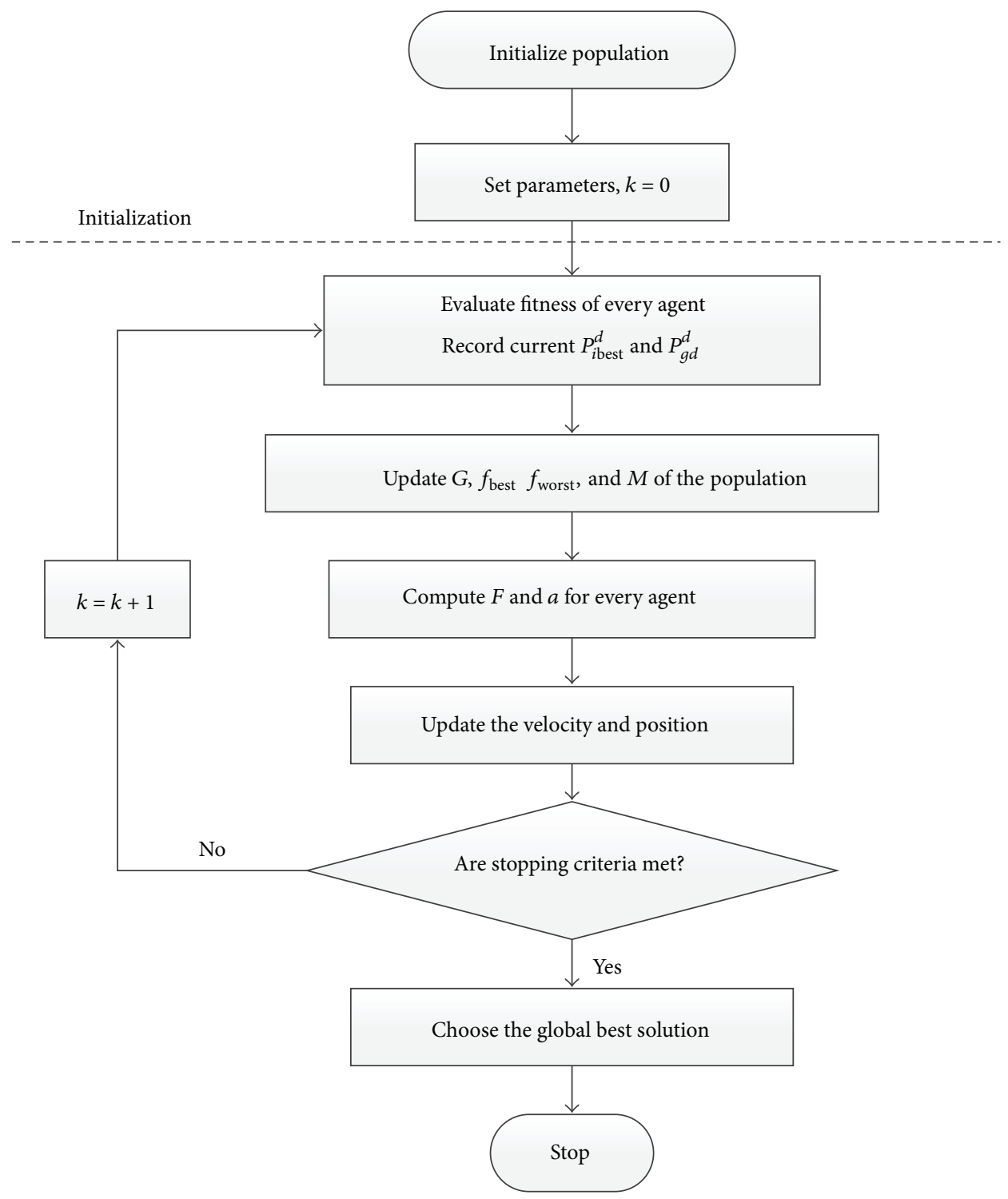

FIGURE 1: Computational flow of the GSAPSO algorithm.

TABLE 1: System data of test systems.

\begin{tabular}{lccc}
\hline Variables & IEEE14-bus system & IEEE30-bus system & IEEE57-bus system \\
\hline Active power of generators (MW) & 272.39 & 289.23 & 1278.66 \\
Reactive power of generators (MVAr) & 82.44 & 139.10 & 321.08 \\
Active power demands (MW) & 259.00 & 283.40 & 1250.80 \\
Reactive power demands (MVAr) & 73.50 & 126.20 & 336.40 \\
Active power losses (MW) & 13.393 & 5.832 & 27.864 \\
Reactive power losses (MVAr) & 54.54 & 30.23 & 121.67 \\
\hline
\end{tabular}

and Table 3 lists the settings of control variables. This network consists of 20 branches, 3 transformers, and 1 capacitor bank. The capacitor bank is set at bus 9. The 3 transformers are connected to branches 4-7, 4-9, and 5-6. In the 14 buses of this test system, bus 1 is the swing bus, 2, 3, 6, and 8 are regarded as the PV buses, and the remaining 9 are the PQ buses.
5.1.2. IEEE30-Bus System. The IEEE30-bus test system is taken as the second test system, whose detailed data is given in $[24,25]$. As shown in Figure 3, this network consists of 41 branches, 4 transformers which are connected to branches 69, 6-10, 4-12, and 28-27, and 9 capacitor banks which are set at buses $10,12,15,17,20,21,23,24$, and 29. This test system has 


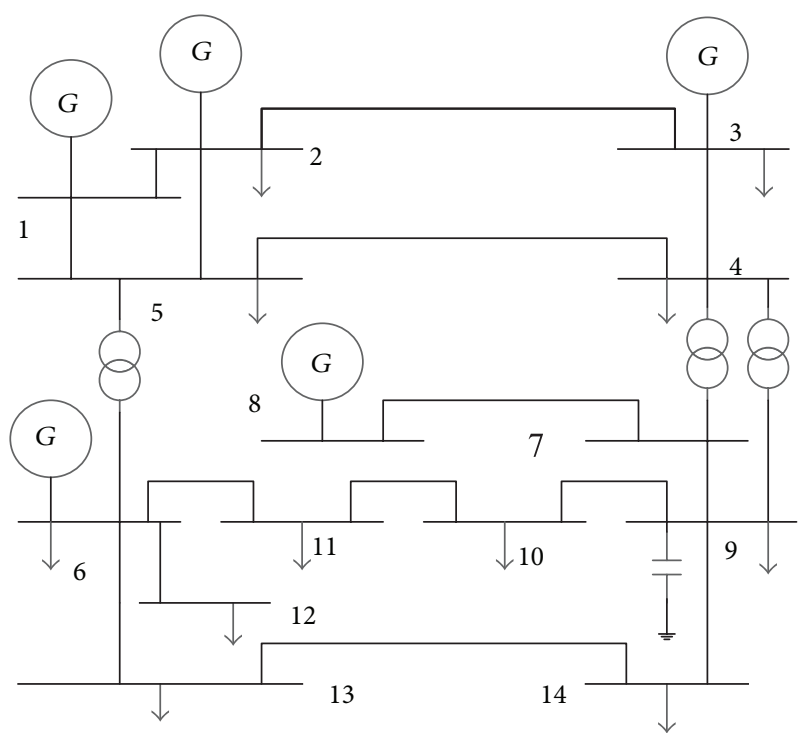

FIGURE 2: The single-line diagram of IEEE14-bus test system.

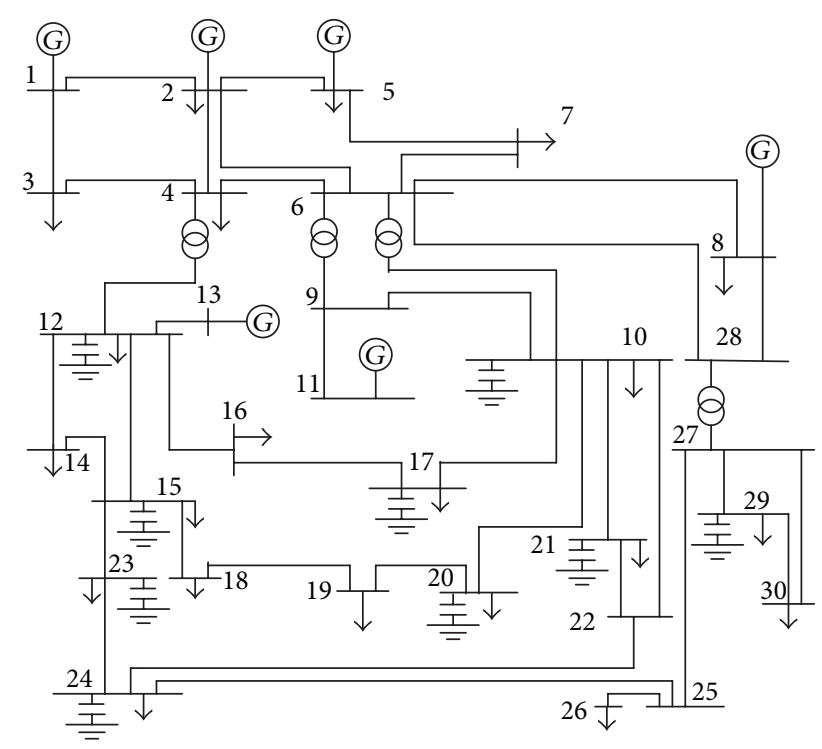

FIGURE 3: The single-line diagram of IEEE30-bus test system.

TABLE 2: Limits of reactive power of generators in IEEE14-bus test system.

\begin{tabular}{lcc}
\hline Bus number & $Q_{\text {gimax }}($ MVAr $)$ & $Q_{\text {gimin }}($ MVAr $)$ \\
\hline 1 & 10 & 0 \\
2 & 50 & -40 \\
3 & 40 & 0 \\
6 & 24 & -6 \\
8 & 24 & -6
\end{tabular}

30 buses. Bus 1 is the swing bus, 2, 5, 8, 11, and 13 are taken as $\mathrm{PV}$ buses, and the remaining 24 are the PQ buses. The reactive power limits of generators are seen in Table 4, and Table 5 lists the settings of control variables.

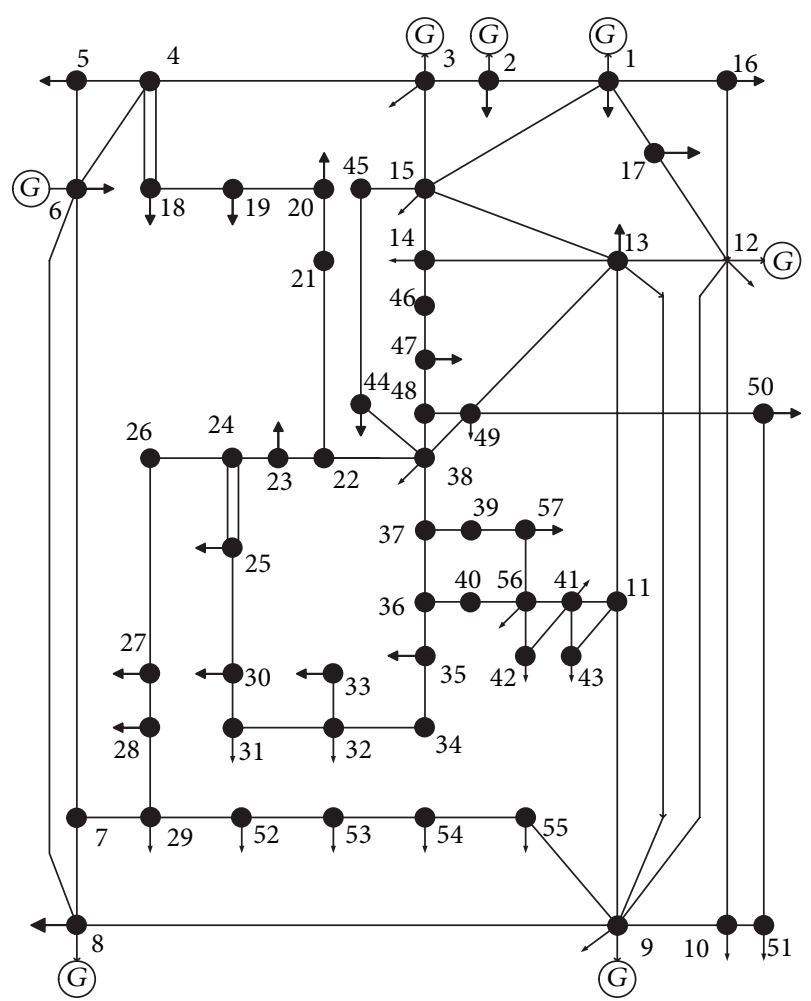

FIGURE 4: The single-line diagram of IEEE57-bus test system.

TABLE 3: Settings of control variables in IEEE14-bus test system.

\begin{tabular}{lccc}
\hline- & Max. & Min. & Step \\
\hline$V_{g}$ (p.u.) & 1.1 & 0.9 & Continuous \\
$T$ & 1.1 & 0.9 & 0.02 \\
$Q_{c}$ (p.u.) & 0.05 & 0 & 0.005 \\
\hline
\end{tabular}

TABLE 4: Limits of reactive power of generators in IEEE30-bus test system.

\begin{tabular}{lcc}
\hline Bus number & $Q_{i \max }($ MVAr $)$ & $Q_{i \min }($ MVAr $)$ \\
\hline 1 & 200 & -20 \\
2 & 100 & -20 \\
5 & 80 & -15 \\
8 & 60 & -15 \\
11 & 50 & -10 \\
13 & 60 & -15 \\
\hline
\end{tabular}

5.1.3. IEEE57-Bus System. In order to verify the applicability of the proposed GSAPSO algorithm for the larger scale system, it has been applied in the IEEE57-bus system whose detailed data is given in [23]. As shown in Figure 4, the network has 80 branches, 17 transformers, and 3 capacitor banks which are set at buses 8,25 , and 53 . The 17 transformers are connected to branches 4-18, 4-18, 21-20, 24-25, 24-25, 2426, 7-29, 34-32, 11-41, 15-45, 14-46, 10-51, 13-49, 11-43, 40$56,39-57$, and $9-55$. In the 57 buses of this system, bus 1 is 


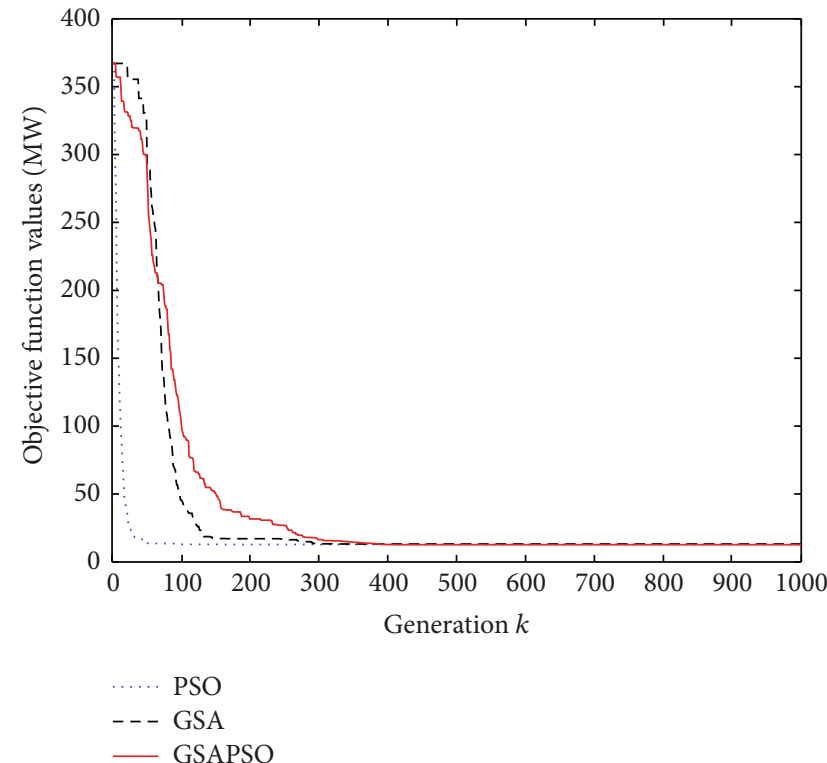

(a)

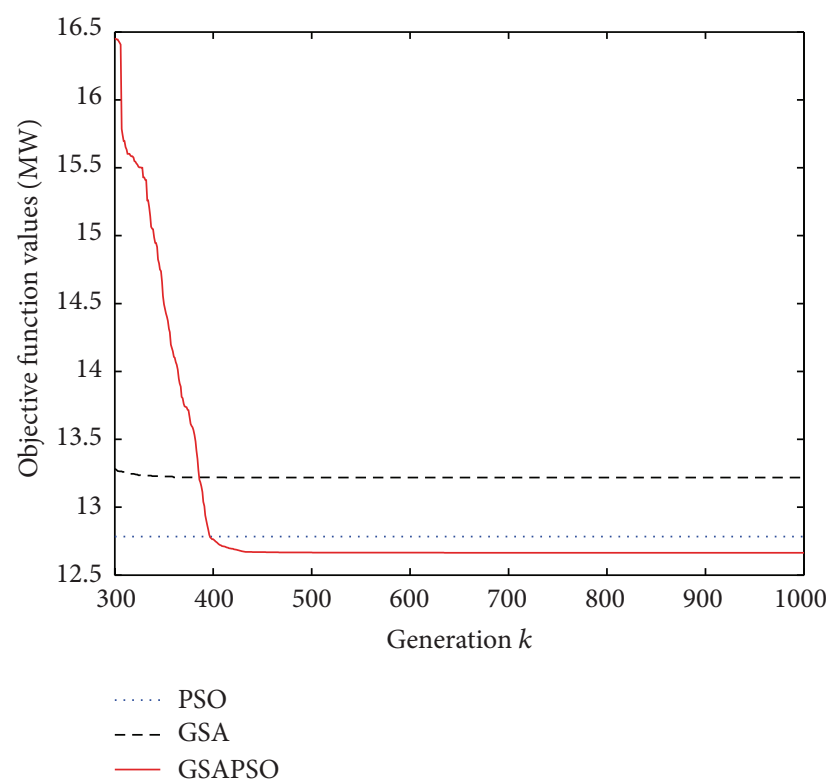

(b)

FIGURE 5: The average convergence curves of different algorithms for IEEE14-bus test system.

TABLE 5: Settings of control variables in IEEE30-bus test system.

\begin{tabular}{lccc}
\hline- & Max. & Min. & Step \\
\hline$V_{g}$ (p.u.) & 1.1 & 0.9 & Continuous \\
$T$ & 1.1 & 0.9 & 0.02 \\
$Q_{c}$ (p.u.) & 0.05 & 0 & 0.005 \\
\hline
\end{tabular}

TABLE 6: Limits of reactive power of generators in IEEE57-bus test system.

\begin{tabular}{lcc}
\hline Bus number & $Q_{i \max }(\mathrm{MVAr})$ & $Q_{i \min }(\mathrm{MVAr})$ \\
\hline 1 & 200 & -140 \\
2 & 130 & -17 \\
3 & 120 & -10 \\
6 & 55 & -8 \\
8 & 200 & -170 \\
9 & 70 & -3 \\
12 & 240 & -150 \\
\hline
\end{tabular}

the swing bus, 2, 3, 6, 8, 9, and 12 are taken as PV buses, and the remaining 50 are PQ buses. The modified reactive power limits of generators are listed in Table 6 , and the settings of control variables are seen in Table 7.

5.2. Parameter Settings. Based on the reduplicative experiments, we find that the smaller population size of particles cannot guarantee the diversity of particle, and the larger population size of particles increases the computational complexity and total computing time. Therefore, by synthesizing each kind of factor, the parameter settings of different algorithms for test systems are listed in Table 8.
TABLE 7: Settings of control variables in IEEE57-bus test system.

\begin{tabular}{lccc}
\hline- & Max. & Min. & Step \\
\hline$V_{g}$ (p.u.) & 1.1 & 0.9 & Continuous \\
$T$ & 1.1 & 0.9 & 0.01 \\
$Q_{c 1}$ (p.u.) & 0.2 & 0.0 & 0.005 \\
$Q_{c 2}$ (p.u.) & 0.18 & 0.0 & 0.006 \\
$Q_{c 3}$ (p.u.) & 0.18 & 0.0 & 0.006 \\
\hline
\end{tabular}

5.3. Simulation Results and Comparison. GSA, PSO, and GSAPSO algorithms, respectively, run 30 times for the abovementioned three test systems. The comparisons of average convergence curves for these test systems are, respectively, shown as Figures 5-7. The distributions of the results for the test systems are shown in Figures 8-10, respectively. The best objective function values and solutions of different algorithms for test systems as well as CPU times are listed in Tables 9-15. Besides, the power loss reduction rates $\left(P_{\text {save }}\right)$ of the compared algorithms are also seen in Tables 9-11.

As seen in Figures 5-7, the proposed GSAPSO can obtain better searching effect than PSO and GSA. For example, in IEEE14-bus test system, PSO, GSA, and GSAPSO get converged, respectively, at about the 20th, 120th, and 300th iteration, which indicates PSO and GSA easily get into the local optimum. And in test systems, compared with PSO and GSA, the proposed GSAPSO can find the solution with lower power losses. In Tables 9-11, the power loss reduction rates of GSAPSO in IEEE14-bus, IEEE30-bus, and IEEE57-bus test systems are $7.05 \%, 17.69 \%$, and $19.51 \%$, respectively, which denotes that the proposed GSAPSO method has a better optimum effect for ORPD problem than other modern artificial intelligence-based techniques including conventional 
TABLE 8: Parameter settings.

\begin{tabular}{|c|c|c|c|c|c|c|c|c|c|}
\hline \multirow{2}{*}{ Parameter } & \multicolumn{3}{|c|}{ IEEE14-bus system } & \multicolumn{3}{|c|}{ IEEE30-bus system } & \multicolumn{3}{|c|}{ IEEE57-bus system } \\
\hline & GSA & PSO & GSAPSO & GSA & PSO & GSAPSO & GSA & $\mathrm{PSO}$ & GSAPSO \\
\hline$N$ & 30 & 30 & 30 & 30 & 30 & 30 & 30 & 30 & 30 \\
\hline$k_{\max }$ & 1000 & 1000 & 1000 & 1000 & 1000 & 1000 & 1000 & 1000 & 1000 \\
\hline$G_{0}$ & 100 & - & 100 & 100 & - & 100 & 100 & - & 100 \\
\hline$\alpha$ & 20 & - & 20 & 20 & - & 20 & 20 & - & 20 \\
\hline$c_{1}$ & - & 2 & 2 & - & 2 & 2 & - & 2 & 2 \\
\hline$c_{2}$ & - & 2 & 2 & - & 2 & 2 & - & 2 & 2 \\
\hline
\end{tabular}

TABLE 9: Comparison of power losses of different algorithms for IEEE14-bus test systems.

\begin{tabular}{|c|c|c|c|c|c|}
\hline Compared item & SARGA [8] & DE [9] & GSA & $\mathrm{PSO}$ & GSAPSO \\
\hline Initial $P_{\text {Loss }}(\mathrm{MW})$ & 13.49 & 13.49 & 13.393 & 13.393 & 13.393 \\
\hline Best $P_{\text {Loss }}(\mathrm{MW})$ & 13.216 & 13.239 & 12.64782 & 12.46522 & 12.44901 \\
\hline$P_{\text {save }}(\%)$ & 2.03 & 1.86 & 5.56 & 6.92 & 7.05 \\
\hline
\end{tabular}

TABLE 10: Comparison of power losses of different algorithms for IEEE30-bus test systems.

\begin{tabular}{lccccc}
\hline Compared item & DE [9] & HAS [26] & GSA & PSO & GSAPSO \\
\hline Initial $P_{\text {Loss }}(\mathrm{MW})$ & 5.660 & 5.934 & 5.832 & 5.832 & 5.832 \\
Best $P_{\text {Loss }}(\mathrm{MW})$ & 5.011 & 4.902 & 5.009544 & 4.915779 & 4.800533 \\
$P_{\text {save }}(\%)$ & 11.47 & 17.39 & 14.10 & 15.71 & 17.69 \\
\hline
\end{tabular}

TABLE 11: Comparison of power losses of different algorithms for IEEE57-bus test systems.

\begin{tabular}{lccccc}
\hline Compared item & DE [9] & SOA [27] & GSA & PSO & GSAPSO \\
\hline Initial $P_{\text {Loss }}(\mathrm{MW})$ & 27.864 & 28.462 & 27.864 & 27.864 & 27.864 \\
Best $P_{\text {Loss }}(\mathrm{MW})$ & 25.048 & 24.625 & 13.48 & 18.30 & 24.22017 \\
$P_{\text {save }}(\%)$ & 10.11 & 13.48482 & 13.08 & 22.42592 \\
\hline
\end{tabular}

TABLE 12: CPU time of different algorithms for test system (s).

\begin{tabular}{|c|c|c|c|c|c|c|c|c|c|}
\hline \multirow{2}{*}{-} & \multicolumn{3}{|c|}{ IEEE14-bus test system } & \multicolumn{3}{|c|}{ IEEE30-bus test system } & \multicolumn{3}{|c|}{ IEEE57-bus test system } \\
\hline & GSA & $\mathrm{PSO}$ & GSAPSO & GSA & PSO & GSAPSO & GSA & PSO & GSAPSO \\
\hline CPU time & 120.2959 & 124.3201 & 120.7331 & 149.7679 & 150.943 & 149.165 & 253.080 & 255.977 & 251.296 \\
\hline
\end{tabular}

TABLE 13: Best solutions of different algorithms for IEEE14-bus test system.

\begin{tabular}{lccc}
\hline $\begin{array}{l}\text { Control } \\
\text { variables }\end{array}$ & GSA & IEEE14-bus test system & PSO \\
\hline$V_{g 1}$ (p.u.) & 1.100000 & 1.100000 & 1.100000 \\
$V_{g 2}$ (p.u.) & 1.076398 & 1.077022 & 1.076853 \\
$V_{g 3}$ (p.u.) & 1.052355 & 1.046782 & 1.046118 \\
$V_{g 6}$ (p.u.) & 1.008185 & 1.020621 & 1.025647 \\
$V_{g 8}$ (p.u.) & 1.049006 & 1.071699 & 1.096356 \\
$T_{1}$ & 1.04 & 1.02 & 0.96 \\
$T_{2}$ & 1.02 & 1.00 & 1.1 \\
$T_{3}$ & 1.00 & 1.04 & 1.04 \\
$Q_{c 1}$ (p.u.) & 0.035 & 0.000 & 0.045 \\
\hline
\end{tabular}


TABLE 14: Best solutions of different algorithms for IEEE30-bus test system.

\begin{tabular}{lccc}
\hline $\begin{array}{l}\text { Control } \\
\text { variables }\end{array}$ & GSA & IEEE30-bus test system \\
\hline$V_{g 1}$ (p.u.) & 1.085932 & 1.074271 & GSAPSO \\
$V_{g 2}$ (p.u.) & 1.077857 & 1.065482 & 1.084864 \\
$V_{g 5}$ (p.u.) & 1.043181 & 1.043299 & 1.053589 \\
$V_{g 8}$ (p.u.) & 1.051025 & 1.043186 & 1.053907 \\
$V_{g 11}$ (p.u.) & 1.071938 & 1.030075 & 1.100000 \\
$V_{g 13}$ (p.u.) & 1.021988 & 1.076433 & 1.051037 \\
$T_{1}$ & 0.98 & 1.10 & 1.10 \\
$T_{2}$ & 1.00 & 0.90 & 0.90 \\
$T_{3}$ & 1.02 & 1.10 & 0.96 \\
$T_{4}$ (p.u. & 0.98 & 1.00 & 1.00 \\
$Q_{c 1}$ (p.u.) & 0.030 & 0.050 & 0.050 \\
$Q_{c 2}$ (p.u.) & 0.035 & 0.000 & 0.000 \\
$Q_{c 3}$ (p.u.) & 0.030 & 0.050 & 0.015 \\
$Q_{c 4}$ (p.u.) & 0.005 & 0.050 & 0.050 \\
$Q_{c 5}$ (p.u.) & 0.020 & 0.000 & 0.035 \\
$Q_{c 6}$ (p.u.) & 0.040 & 0.050 & 0.050 \\
$Q_{c 7}$ (p.u.) & 0.030 & 0.050 & 0.050 \\
$Q_{c 8}$ (p.u.) & 0.025 & 0.050 & 0.050 \\
$Q_{c 9}$ (p.u.) & 0.050 & 0.025 & 0.045 \\
\hline
\end{tabular}
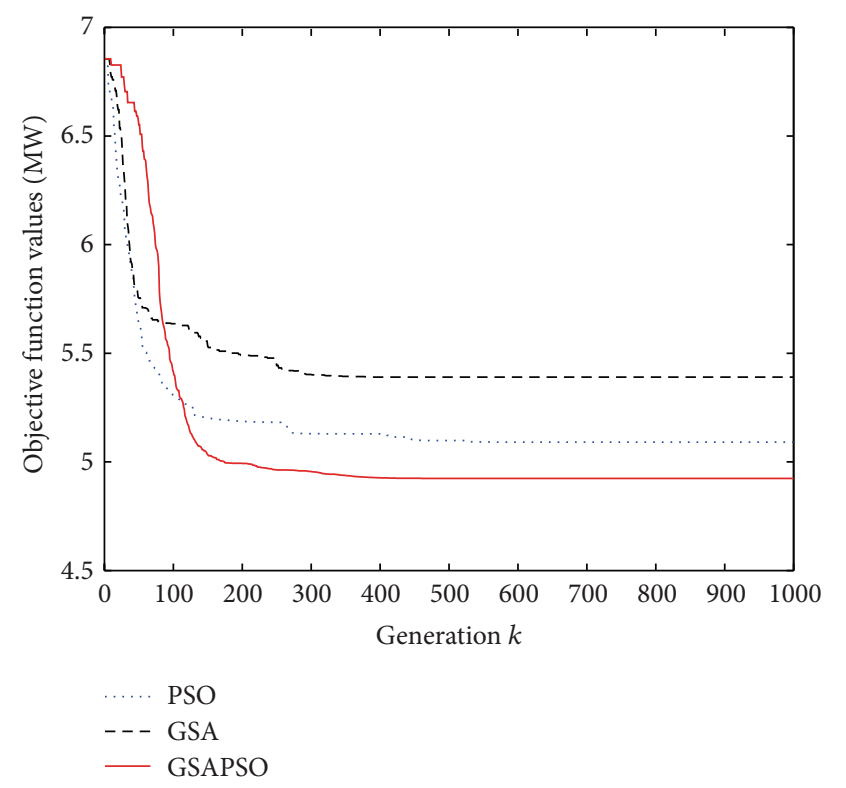

FIGURE 6: The average convergence curves of different algorithms for IEEE30-bus test system.

PSO and GSA algorithms. Furthermore, the CPU times shown in Table 12 make it clear that the run times of three different algorithms are not much different for these test systems, which shows that the effectiveness of the proposed method does not mean the low efficiency. Owning to the higher complexity of IEEE57-bus test system, its CPU time is
TABLE 15: Best solutions of different algorithms for IEEE57-bus test system.

\begin{tabular}{|c|c|c|c|}
\hline \multirow{2}{*}{$\begin{array}{l}\text { Control } \\
\text { variables }\end{array}$} & \multicolumn{3}{|c|}{ IEEE30-bus test system } \\
\hline & GSA & PSO & GSAPSO \\
\hline$V_{g 1}$ (p.u.) & 1.100000 & 1.100000 & 1.100000 \\
\hline$V_{g 2}$ (p.u.) & 1.100000 & 1.100000 & 1.100000 \\
\hline$V_{g 3}$ (p.u.) & 1.089813 & 1.100000 & 1.085288 \\
\hline$V_{g 6}$ (p.u.) & 1.084215 & 1.100000 & 1.080974 \\
\hline$V_{g 8}$ (p.u.) & 1.100000 & 1.100000 & 1.100000 \\
\hline$V_{g 9}$ (p.u.) & 1.084676 & 1.100000 & 1.081252 \\
\hline$V_{g 12}$ (p.u.) & 1.080065 & 1.100000 & 1.075432 \\
\hline$T_{1}$ & 1.10 & 1.10 & 0.90 \\
\hline$T_{2}$ & 1.01 & 1.10 & 1.10 \\
\hline$T_{3}$ & 1.10 & 1.10 & 1.02 \\
\hline$T_{4}$ & 1.10 & 0.90 & 1.10 \\
\hline$T_{5}$ & 0.97 & 1.10 & 0.90 \\
\hline$T_{6}$ & 1.10 & 0.98 & 0.99 \\
\hline$T_{7}$ & 1.10 & 0.99 & 0.99 \\
\hline$T_{8}$ & 0.90 & 0.94 & 0.90 \\
\hline$T_{9}$ & 0.90 & 1.10 & 1.10 \\
\hline$T_{10}$ & 0.98 & 1.01 & 0.97 \\
\hline$T_{11}$ & 0.97 & 1.02 & 0.97 \\
\hline$T_{12}$ & 0.98 & 1.10 & 0.98 \\
\hline$T_{13}$ & 0.94 & 1.02 & 0.94 \\
\hline$T_{14}$ & 1.09 & 1.10 & 0.94 \\
\hline$T_{15}$ & 1.03 & 0.99 & 1.10 \\
\hline$T_{16}$ & 1.10 & 0.97 & 1.01 \\
\hline$T_{17}$ & 1.10 & 1.10 & 1.00 \\
\hline$Q_{c 1}$ (p.u.) & 0.000 & 0.200 & 0.180 \\
\hline$Q_{c 2}$ (p.u.) & 0.156 & 0.180 & 0.108 \\
\hline$Q_{c 3}$ (p.u.) & 0.150 & 0.180 & 0.180 \\
\hline
\end{tabular}

longer than that of IEEE14-bus and IEEE30-bus test systems. Besides, according to Figures $8-10$, it is obvious that the distribution of the results of GSAPSO was more concentrated in a smaller range than that of PSO and GSA, which manifests that the result uniformity of the proposed GSAPSO is better than GSA and PSO.

\section{Conclusions}

GSAPSO is a novel heuristic stochastic optimization algorithm, which combined the memory characteristic of PSO based on the traditional GSA algorithm. The proposed GSAPSO, PSO, and GSA algorithms have been successfully introduced to solve the ORPD problem based on IEEE14bus, IEEE30-bus, and IEEE57-bus test power systems in this paper. The simulation results demonstrate the potential and effectiveness of the proposed GSAPSO approach for solving 


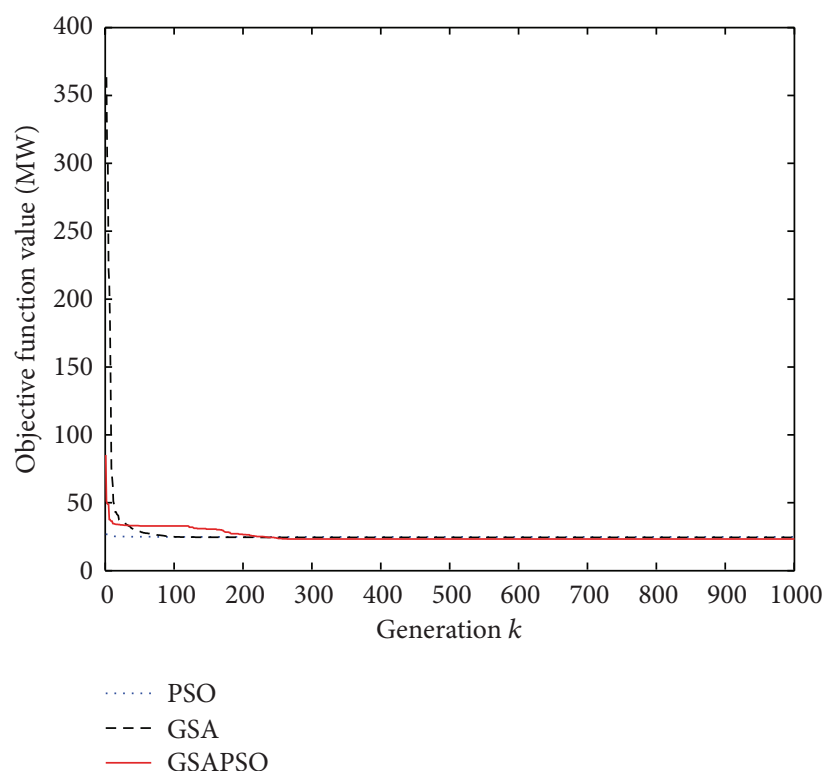

(a)

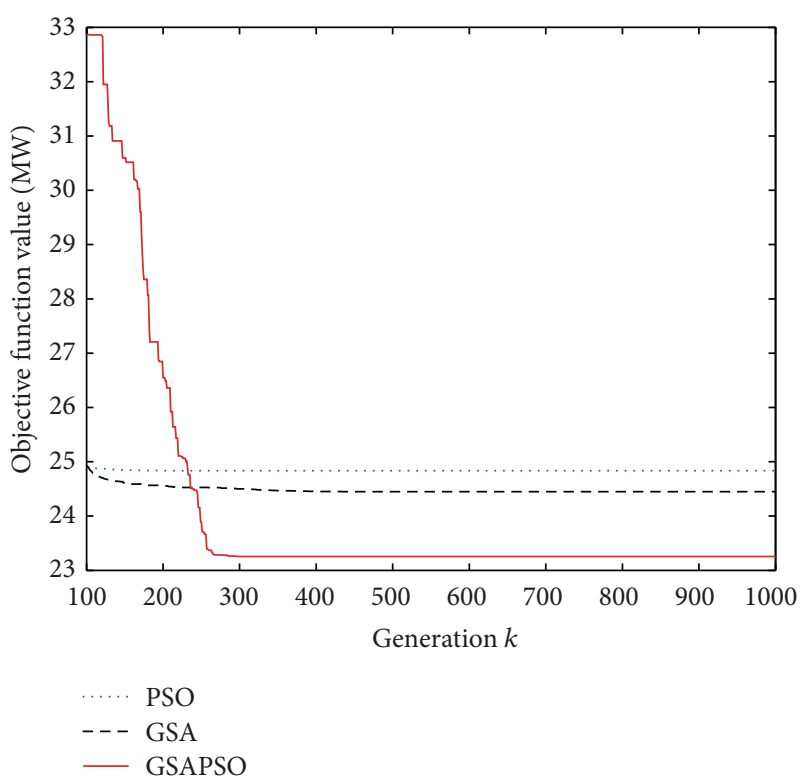

(b)

FIGURE 7: The average convergence curves of different algorithms for IEEE57-bus test system.

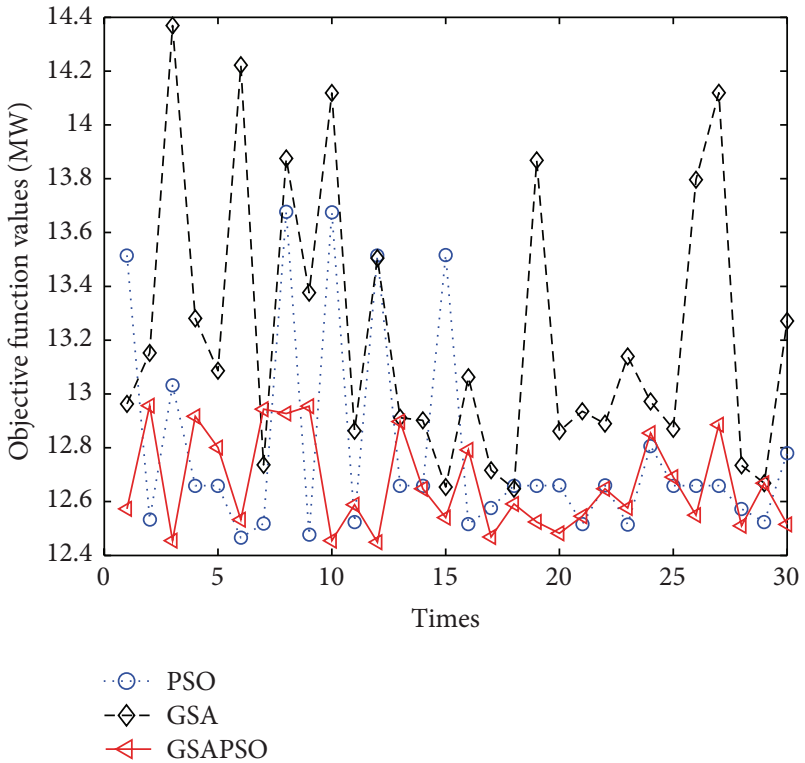

FIGURE 8: The distribution of the results of different algorithms for IEEE14-bus test system.

ORPD problem of power system, especially in the larger IEEE57-bus test system. The proposed GSAPSO method can obtain bigger power loss reduction rates than the compared algorithms, and its results' distribution is more concentrated compared to that of GSA and PSO under the condition that these algorithms take almost the same computing time. Besides, the proposed GSAPSO can avoid falling into the local optimum.

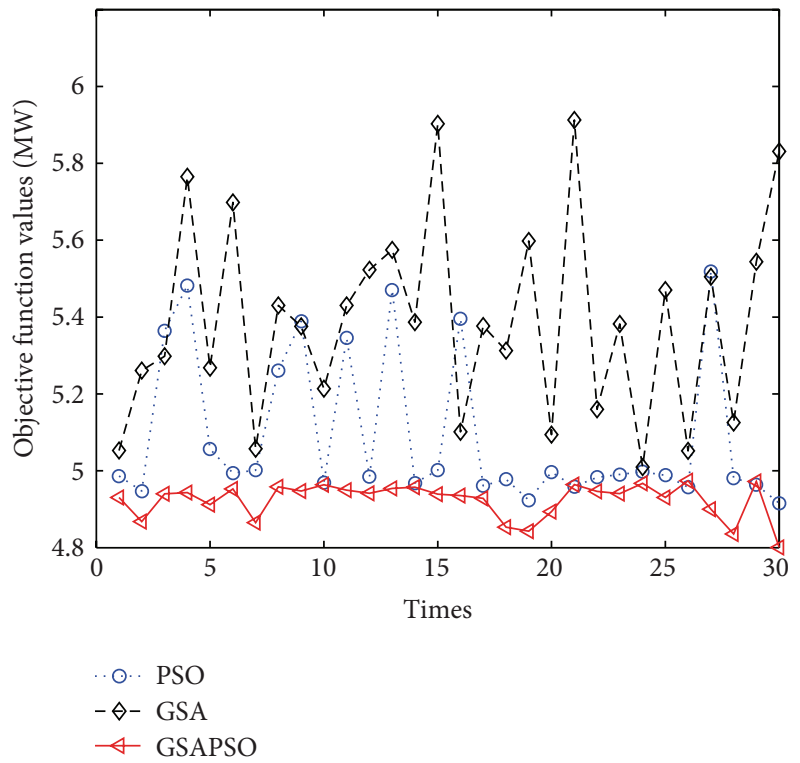

Figure 9: The distribution of the results of different algorithms for IEEE30-bus test system.

\section{Conflict of Interests}

The authors declare that there is no conflict of interests regarding the publication of this paper.

\section{Acknowledgments}

This work was supported by the National Natural Science Foundation of China (nos. 51207064 and 51507024) and 


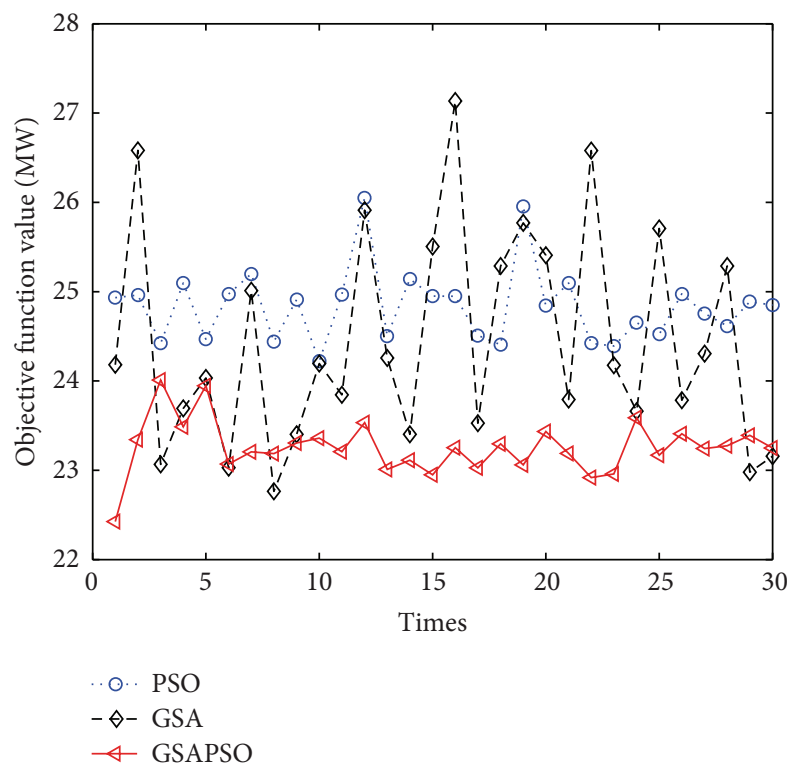

FIGURE 10: The distribution of the results of different algorithms for IEEE57-bus test system.

Science and Technology Research Project of Chongqing Municipal Education Commission (no. KJ1500401).

\section{References}

[1] G. Chen, L. Liu, P. Song, and Y. Du, "Chaotic improved PSObased multi-objective optimization for minimization of power losses and $L$ index in power systems," Energy Conversion and Management, vol. 86, pp. 548-560, 2014.

[2] H. H. Happ, "Optimal power dispatch-a comprehensive survey," IEEE Transactions on Power Apparatus and Systems, vol. 96, no. 3, pp. 841-854, 1977.

[3] V. H. Quintana and M. Santos-Nieto, "Reactive-power dispatch by successive quadratic programming," IEEE Transactions on Energy Conversion, vol. 4, no. 3, pp. 425-435, 1989.

[4] S. Granville, "Optimal reactive dispatch through interior point methods," IEEE Transactions on Power Systems, vol. 9, no. 1, pp. 136-146, 1994.

[5] J. L. M. Ramos, A. G. Expósito, and V. H. Quintana, “Transmission power loss reduction by interior-point methods: implementation issues and practical experience," IEE Proceedings Generation Transmission Distribution, vol. 152, no. 1, pp. 90-98, 2005.

[6] M. R. AlRashidi and M. E. El-Hawary, "Applications of computational intelligence techniques for solving the revived optimal power flow problem," Electric Power Systems Research, vol. 79, no. 4, pp. 694-702, 2009.

[7] B. Zhao, C. X. C. Guo, and Y. J. Cao, "A multiagent-based particle swarm optimization approach for optimal reactive power dispatch," IEEE Transactions on Power Systems, vol. 20, no. 2, pp. 1070-1078, 2005.

[8] P. Subbaraj and P. N. Rajnarayanan, "Optimal reactive power dispatch using self-adaptive real coded genetic algorithm," Electric Power Systems Research, vol. 79, no. 2, pp. 374-381, 2009.

[9] M. Varadarajan and K. S. Swarup, "Differential evolutionary algorithm for optimal reactive power dispatch," International
Journal of Electrical Power and Energy Systems, vol. 30, no. 8, pp. 435-441, 2008.

[10] B. Kanna and S. N. Singh, "Towards reactive power dispatch within a wind farm using hybrid PSO," International Journal of Electrical Power \& Energy Systems, vol. 69, pp. 232-240, 2015.

[11] X. Yuan, B. Ji, Y. Yuan, R. M. Ikram, X. Zhang, and Y. Huang, "An efficient chaos embedded hybrid approach for hydrothermal unit commitment problem," Energy Conversion and Management, vol. 91, pp. 225-237, 2015.

[12] T. Niknam, E. Azadfarsani, and M. Jabbari, "A new hybrid evolutionary algorithm based on new fuzzy adaptive PSO and NM algorithms for Distribution Feeder Reconfiguration," Energy Conversion and Management, vol. 54, no. 1, pp. 7-16, 2012.

[13] S. Liao, C. Cheng, J. Wang, and Z. Feng, "A hybrid search algorithm for midterm optimal scheduling of thermal power plants," Mathematical problems in engineering, vol. 2015, 13 pages, 2015.

[14] E. Rashedi, H. Nezamabadi-pour, and S. Saryazdi, "GSA: a gravitational search algorithm," Information Sciences, vol. 179, no. 13, pp. 2232-2248, 2009.

[15] A. Chatterjee, K. Roy, and D. Chatterjee, "A Gravitational Search Algorithm (GSA) based Photo-Voltaic (PV) excitation control strategy for single phase operation of three phase wind-turbine coupled induction generator," Energy, vol. 74, pp. 707-718, 2014.

[16] M. Eslami, H. Shareef, A. Mohamed, and M. Khajehzadeh, "PSS and TCSC damping controller coordinated design using GSA," Energy Procedia, vol. 14, pp. 763-769, 2012.

[17] S. Duman, U. U. Güvenç, Y. Sönmez, and N. Yörükeren, "Optimal power flow using gravitational search algorithm," Energy Conversion and Management, vol. 59, pp. 86-95, 2012.

[18] J. Kennedy and R. Eberhart, "Particle swarm optimization," in Proceedings of the IEEE International Conference on Neural Networks, vol. 4, pp. 1942-1948, IEEE, Perth, Australia, NovemberDecember 1995.

[19] X. Yuan, L. Wang, and Y. Yuan, "Application of enhanced PSO approach to optimal scheduling of hydro system," Energy Conversion and Management, vol. 49, no. 11, pp. 2966-2972, 2008.

[20] T.-H. Kim, I. Maruta, and T. Sugie, "Robust PID controller tuning based on the constrained particle swarm optimization," Automatica, vol. 44, no. 4, pp. 1104-1110, 2008.

[21] R. Barbieri, N. Barbieri, and K. F. de Lima, "Some applications of the PSO for optimization of acoustic filters," Applied Acoustics, vol. 89, pp. 62-70, 2015.

[22] B. Shaw, V. Mukherjee, and S. P. Ghoshal, "Solution of reactive power dispatch of power systems by an opposition-based gravitational search algorithm," International Journal of Electrical Power \& Energy Systems, vol. 55, pp. 29-40, 2014.

[23] R. D. Zimmerman, C. E. Murillo-Sánchez, and D. Gan, "MATPOWER: a matable power system simulation package," http://www.pserc.cornell.edu/matpower/.

[24] O. Alsac and B. Stott, "Optimal load flow with steady-state security," IEEE Transactions on Power Apparatus and Systems, vol. 93, no. 3, pp. 745-751, 1974.

[25] K. Y. Lee, Y. M. Park, and J. L. Ortiz, "A united approach to optimal real and reactive power dispatch," IEEE Transactions on Power Apparatus and Systems, vol. 104, no. 5, pp. 1147-1153, 1985.

[26] A. H. Khazali and M. Kalantar, "Optimal reactive power dispatch based on harmony search algorithm," International Journal of Electrical Power and Energy Systems, vol. 33, no. 3, pp. 684-692, 2011. 
[27] C. Dai, W. Chen, Y. Zhu, and X. Zhang, "Reactive power dispatch considering voltage stability with seeker optimization algorithm," Electric Power Systems Research, vol. 79, no. 10, pp. 1462-1471, 2009. 


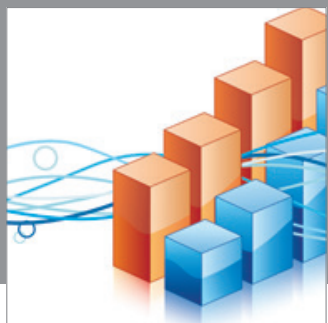

Advances in

Operations Research

mansans

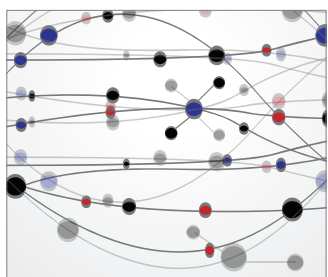

The Scientific World Journal
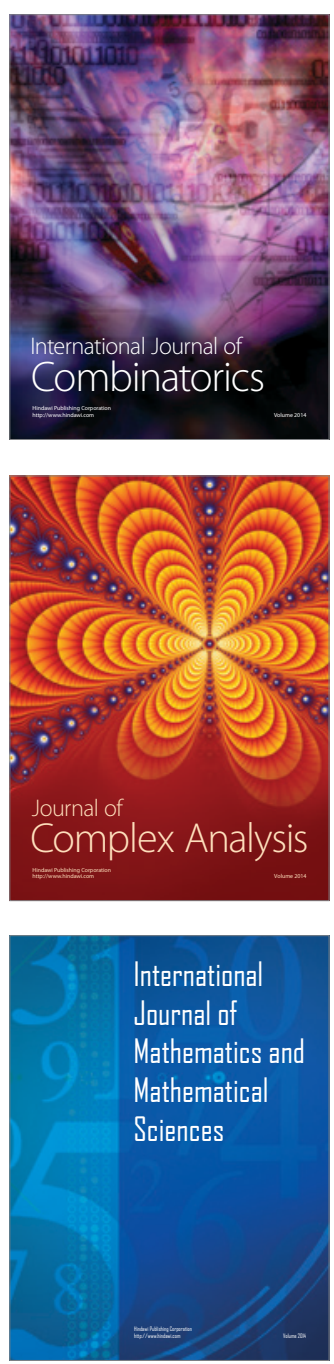
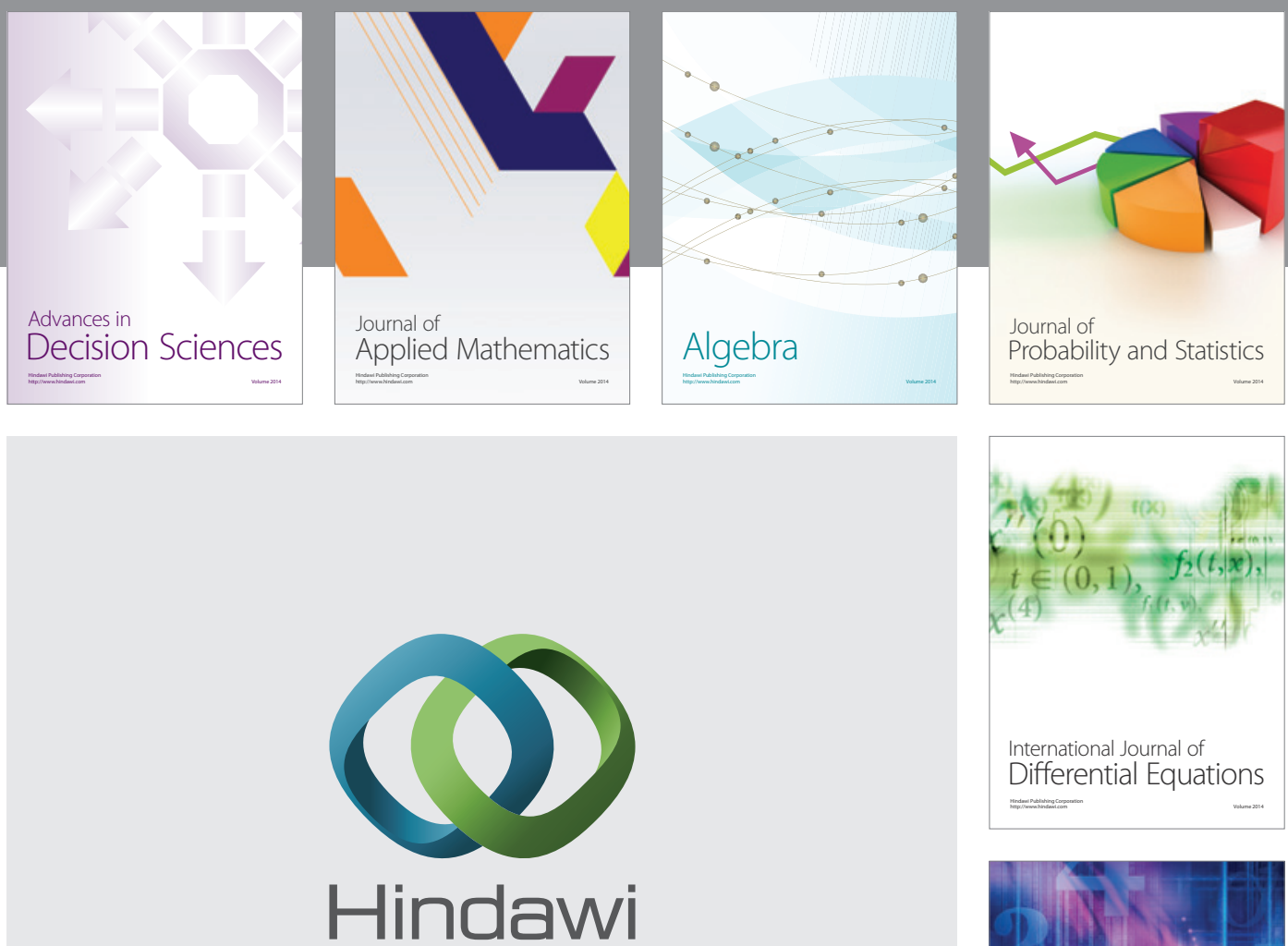

Submit your manuscripts at http://www.hindawi.com
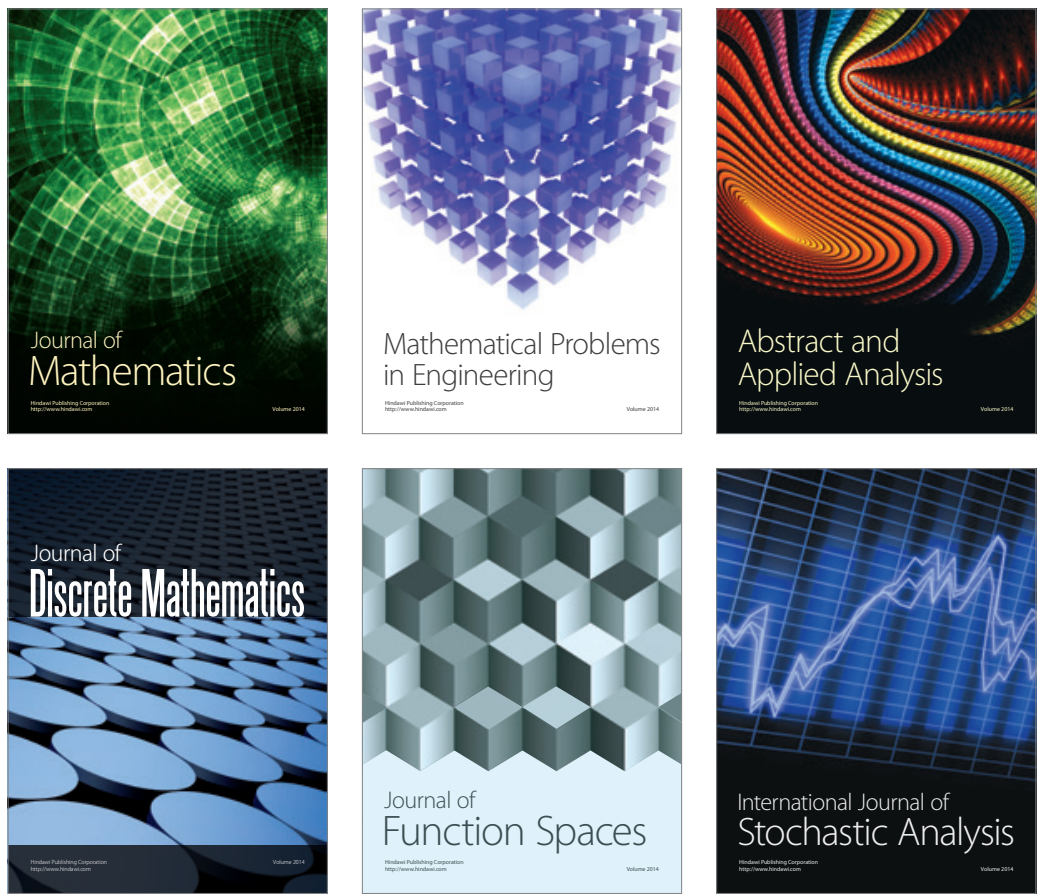

Journal of

Function Spaces

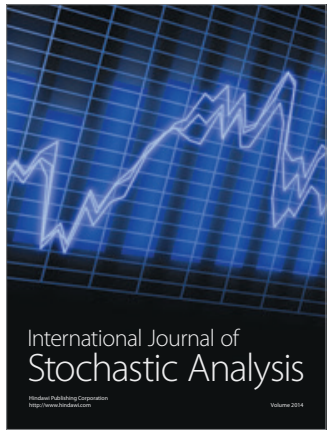

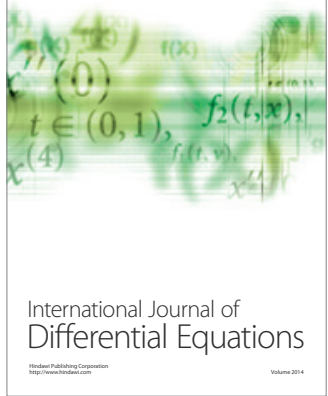
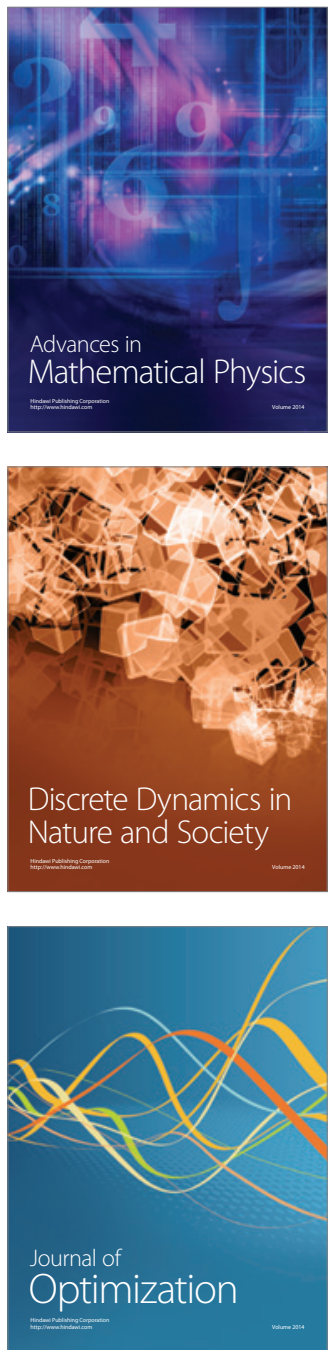